\title{
Restoring facial symmetry through non-surgical cosmetic procedures after permanent facial paralysis: a case report
}

\author{
Ali Sahan ${ }^{1}$, Funda Tamer²凶
}

\begin{abstract}
Facial nerve paralysis can occur due to infection, inflammation, trauma, surgery, and tumors. It leads to facial asymmetry, impaired oral competence, articulation deficits, and psychological problems. Treatment options include physical therapy, static slings, nerve and muscle transfers, blepharoplasty, brow lift, and chemodenervation with botulinum toxin. We report the case of a 66-year-old Caucasian female with permanent facial paralysis following middle ear surgery. The facial asymmetry was treated successfully with botulinum toxin A injection, hyaluronic acid dermal filler injection, and a thread-lift procedure.
\end{abstract}

Keywords: cosmetic procedures, facial paralysis

Received: 8 January 2017 | Returned for modification: 9 February 2017 | Accepted: 16 February 2017

\section{Introduction}

The facial nerve innervates the muscles of facial expression. Dysfunction of the facial nerve is called facial paralysis. Facial paralysis may cause various psychological and functional problems. Bell's palsy is the most common cause of facial paralysis. Bell's palsy is usually transient and it heals spontaneously. However, in a few patients it can be persistent (1). Moreover, surgical trauma can cause facial nerve paralysis. In our patient, facial nerve paralysis occurred 3 days after a middle ear surgery. The treatment requires a multidisciplinary approach including pharmacological and physical therapy or surgical procedures (2). Chronic facial paralysis that lasts more than 2 years results in muscle atrophy. Therefore, dynamic reanimation including muscle transfer procedures, static procedures such as oculoplasty, facial sling suspension, endoscopic and open facelift, brow lift, and lip shortening or thickening may be performed $(2,3)$. Furthermore, botulinum toxin injections are used to treat involuntary muscle spasms and facial asymmetry. The orbicularis oculi, corrugator supercilii, platysma, mentalis, and buccinator are the most preferred muscles for injection (4).

\section{Case report}

A 66-year-old Caucasian female patient was admitted for further clinical evaluation of facial asymmetry. The patient had had unilateral facial nerve paralysis for the previous 4 years following middle ear surgery. Two years earlier, the patient had undergone surgical blepharoplasty to lift the left upper eyelid because of ptosis. The patient was otherwise healthy. However, she had cosmetic concerns. Physical examination revealed a distinct asymmetrical facial appearance. A total dose of 6.5 units of botulinum toxin A (1.5, two, and three units into three sites from lateral to medial) was injected intramuscularly into the right part of the frontal muscle. A total dose of four units of botulinum toxin A was injected into the right levator labii superior muscle, two units into the right levator anguli oris muscle, two units into the left depressor anguli oris muscle, and two units into the right inferior
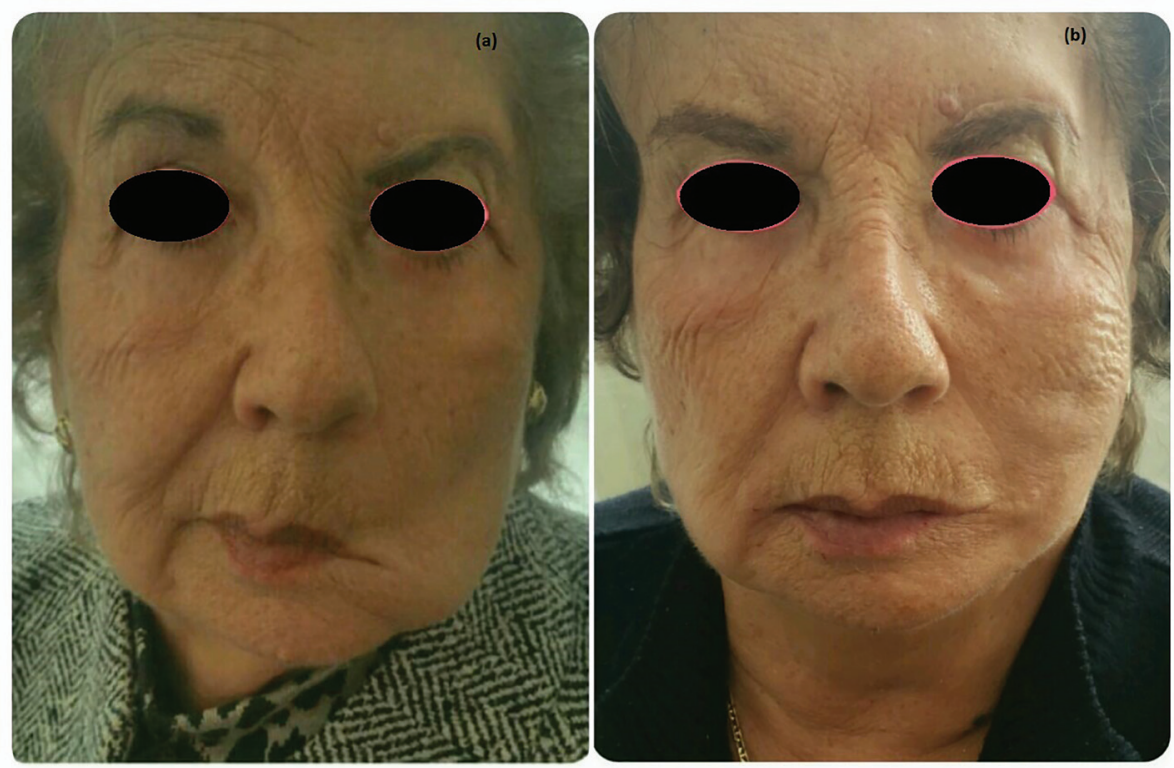

Figure 1 ( Before a) and 4 weeks after b) treatment.

120ctor Al-Sa Aesthetic \& Cosmetic Dermatology Clinic, Ankara, Turkey. ${ }^{2}$ Medical Park Hospital, Department of Dermatology, Ankara, Turkey. $\bowtie$ Corresponding author: fundatmr@yahoo.com 
orbicularis oris muscle. Furthermore, $1 \mathrm{cc}$ of $20 \mathrm{mg} / \mathrm{ml}$ hyaluronic acid (HA) dermal filler was injected to enhance the left medial and middle cheek fat; $0.5 \mathrm{cc}$ of $20 \mathrm{mg} / \mathrm{ml} \mathrm{HA}$ was injected for reconstruction of the left zygomatic arch; $0.5 \mathrm{cc}$ of $20 \mathrm{mg} / \mathrm{ml} \mathrm{HA}$ was used to contour the left nasolabial fold, and $0.2 \mathrm{cc}$ of $20 \mathrm{mg} / \mathrm{ml}$ HA was injected to raise the left eyebrow. Finally, thread lifting was performed to the left side of the face using a cannula cog to lift the superior and inferior jowl fat, nasolabial fold, and middle cheek fat. An excellent cosmetic outcome was achieved 4 weeks after treatment (Figs. 1a-b).

\section{Discussion}

Facial paralysis can result in aesthetic, psychological, and functional problems. The patient characteristics, medical history, causes, and duration of facial paralysis should be evaluated for appropriate treatment (1). Sadiq et al. treated 14 patients with facial nerve paralysis using botulinum toxin. The injection sites were the contralateral lower facial muscles (elevator and depressor angularis oris). They aimed to weaken the normal muscles for a symmetrical appearance. The symmetry was evaluated with the ratio of mean muscle activity. Botulinum toxin injection improved facial symmetry; however, the effect was more noticeable in younger patients (5). Navarrete et al. used Silhouette Sutures to improve facial asymmetry after facial palsy. The first patient was

\section{References}

1. Leckenby J, Grobbelaar A. Smile restoration for permanent facial paralysis. Arch Plast Surg. 2013;40:633-8.

2. Mehta RP. Surgical treatment of facial paralysis. Clin Exp Otorhinolaryngol. 2009;2:1-5.

3. Ibrahim AM, Rabie AN, Kim PS, Medina M, Upton J, Lee BT, et al. Static treatment modalities in facial paralysis: a review. J Reconstr Microsurg. 2013;29:223-32

4. Mehdizadeh OB, Diels J, White WM. Botulinum toxin in the treatment of facial paralysis. Facial Plast Surg Clin North Am. 2016;24:11-20. a 79-year-old female who had facial paralysis as a result of otic tuberculosis. The second patient was a 77-year-old female who had facial paralysis after total parotidectomy. They reported good cosmetic and functional outcomes in both patients with Silhouette Sutures (6).

In this case, we performed non-surgical cosmetic procedures in the dermatology outpatient clinic. Botulinum toxin injection, dermal filler injection, and thread lifting were all performed during the same treatment session. In spite of the long disease duration and advanced age, a satisfactory treatment result was obtained. The patient stated that the treatment had markedly improved her self-esteem.

Our patient had a 4-year history of facial paralysis. She had cosmetic concerns; however, she did not have a significant functional impairment. The patient had had a previous unsatisfactory eyelid surgery. Therefore, we preferred these non-surgical procedures to improve facial asymmetry. To the best of our knowledge, this is the first case of facial asymmetry successfully treated using botulinum toxin A injection, hyaluronic acid dermal filler injection, and a thread-lift procedure.

In conclusion, facial paralysis requires a multidisciplinary therapy. However, non-surgical and minimally invasive cosmetic procedures may be the preferred treatment option. Dermatologists seem to be acquiring a more important role in the treatment of permanent facial paralysis.
5. Sadiq SA, Khwaja S, Saeed SR. Botulinum toxin to improve lower facial symmetry in facial nerve palsy. Eye (Lond). 2012;26:1431-6.

6. Navarrete ML, Palao R, Torrent L, Fuentes JF, Gonzalez M. Facial asymmetry correction in facial palsy patients with Silhouette Sutures. Int J Clin Med. 2012;3:55-9. 